\title{
Intersectin-1s deficiency in pulmonary pathogenesis
}

Niranjan Jeganathan ${ }^{1 *}$, Dan Predescu ${ }^{2}$ and Sanda Predescu ${ }^{3}$

\begin{abstract}
Intersectin-1s (ITSN-1s), a multidomain adaptor protein, plays a vital role in endocytosis, cytoskeleton rearrangement and cell signaling. Recent studies have demonstrated that deficiency of ITSN-1s is a crucial early event in pulmonary pathogenesis. In lung cancer, ITSN-1s deficiency impairs Eps8 ubiquitination and favors Eps8-mSos1 interaction which activates Rac1 leading to enhanced lung cancer cell proliferation, migration and metastasis. Restoring ITSN-1s deficiency in lung cancer cells facilitates cytoskeleton changes favoring mesenchymal to epithelial transformation and impairs lung cancer progression. ITSN-1s deficiency in acute lung injury leads to impaired endocytosis which leads to ubiquitination and degradation of growth factor receptors such as Alk5. This deficiency is counterbalanced by microparticles which, via paracrine effects, transfer Alk5/TGFBRII complex to non-apoptotic cells. In the presence of ITSN-1s deficiency, Alk5-restored cells signal via Erk1/2 MAPK pathway leading to restoration and repair of lung architecture. In inflammatory conditions such as pulmonary artery hypertension, ITSN-1s full length protein is cleaved by granzyme B into EH $H_{\text {TSN }}$ and SH3A-E ITSN fragments. The EH ITSN fragment leads to pulmonary cell proliferation via activation of p38 MAPK and Elk-1/c-Fos signaling. In vivo, ITSN-1s deficient mice transduced with $E_{\text {ITSN }}$ plasmid develop pulmonary vascular obliteration and plexiform lesions consistent with pathological findings seen in severe pulmonary arterial hypertension. These novel findings have significantly contributed to understanding the mechanisms and pathogenesis involved in pulmonary pathology. As demonstrated in these studies, genetically modified ITSN-1s expression mouse models will be a valuable tool to further advance our understanding of pulmonary pathology and lead to novel targets for treating these conditions.
\end{abstract}

Keywords: Intersectin-1s, Pulmonary arterial hypertension, Lung cancer, Acute lung injury, Eps8, mSos1, MAPK, Rac1

\section{Introduction}

Intersectins (ITSNs) belong to the family of adaptor proteins [1]. Adaptor proteins, also known as scaffold proteins, mediate the interaction between receptors and signal transduction pathways by functioning as platforms for the assembly of multiple protein signaling complexes [2, 3]. Adaptor proteins lead to specificity in signaling via their sequence of protein domains/motifs, subcellular localization and their proximity to binding proteins [2, 3]. Thus, adaptor proteins play a crucial role in cell signaling in a spatial and temporal fashion, and regulate many important cellular processes including proliferation, differentiation, cell cycle control, cell survival and migration $[2,3]$. Aberrant expression of adaptor proteins is implicated in numerous diseases [3].

\footnotetext{
* Correspondence: Niranjan_Jeganathan@Rush.edu

'Rush University Medical Center, Chicago, IL 60612, USA

Full list of author information is available at the end of the article
}

ITSNs have a unique multi-domain structure each with a distinct ligand preference [4]. ITSNs were initially only associated with the regulation of endocytosis [5]. Subsequent studies have revealed a more complex role for these proteins in the regulation of cell signaling and cytoskeleton rearrangement $[1,6-10]$. Recent studies implicate ITSNs, especially the transcript intersectin-1 short (ITSN$1 \mathrm{~s})$, in the pathogenesis of several pulmonary diseases [11-16]. Given these novel findings, this review article will provide a comprehensive overview of ITSN-1s' regulation of biochemical pathways and its clinical implications in pulmonary pathology.

\section{Background}

The sequence and initial characterization of ITSN was first reported in Xenopus in 1998 by Yamabhai et al. [17]. They reported a 1270-amino acid long protein containing two Eps15 homology (EH) domains, a central coiled- 
coiled region, and five Src homology 3 (SH3) domains (Fig. 1). The protein was named ITSN because of its potential to bring together $\mathrm{EH}$ and $\mathrm{SH} 3$ domain binding proteins into a macromolecular complex [17]. ITSN was the first protein reported with both $\mathrm{EH}$ and $\mathrm{SH} 3$ domains which interacts with asparagine-proline-phenylalanine (NPF) and proline-rich (PXXP) motifs of the binding proteins, respectively [18].

Shortly thereafter, human ITSN was identified by Guipponi et al. [19]. ITSN exists with a high degree of similarity in a number of higher eukaryotes [20]. There are two ITSN genes in humans, ITSN-1 and ITSN-2, located on chromosome 21 and 2 respectively $[19,21]$. ITSN-1 and ITSN-2 share identical domain structure and greater than $50 \%$ sequence identity $[22,23]$. The human ITSN gene produces two main ITSN transcript mRNAs, short (ITSN-s) and long (ITSN-l), due to alternative splicing; human ITSN-s is 5.3-kilobases and ITSN-1 is 15-kilobases [19]. Both ITSN-s and ITSN-l contain two EH and five SH3 domains $[19,21]$. In addition, ITSN-1 encodes for a Dbl homology (DH), a Pleckstrin (PH) and a C2 domain [19], Fig. 1. The EH domains bind to proteins associated with endocytosis $[4,17]$. SH3 domains interact with proteins implicated in cell signaling and cytoskeleton organization $[6,7,11,13,24,25]$. The DH domains promote guaninenucleotide exchange on Rho, $\mathrm{PH}$ domains mediate interactions with inositol phospholipid and $\mathrm{C} 2$ domains mediate Ca2-dependent phospholipid binding [26].

ITSNs are widely distributed in human tissues, however the expression of ITSN isoforms is tissue-dependent; ITSN-1 1 is specific to the brain and is absent in lung tissue whereas ITSN-1s, ITSN-2 $\mathrm{s}$ and ITSN-2 1 are expressed ubiquitously [19, 21, 23]. Alternative splicing plays a major role in the regulation of ITSN gene expression, function and tissue specificity $[4,27]$. In addition to the major splice variants there is a number of minor splice variants of ITSN-1s protein which facilitate tissue specific interactions $[4,19,21]$. This is illustrated in the fact that ITSN-1s preferentially interacts with mSos1 and $\mathrm{Cbl}$ in most tissues including lung $[6,11,13,28]$. However, in brain tissue, a splicing of microexon 20 within the SH3A domain of ITSN-1s (resulting in inclusion of 5 additional amino acids) leads to reduced binding to $\mathrm{mSos} 1$ and $\mathrm{Cbl}$, and enhanced interaction with CdGAP [29]. This transcript was conserved in numerous other eukaryotes examined. Additional splicing events have also been reported with ITSN-1s [4].

ITSN-1s is present in all subcellular compartments [8]. At the plasma membrane it is present in caveolae and clathrin-coated pits $[8,13,26]$. Activation of receptor tyrosine kinases (RTK) relocates ITSN-1s to the plasma membrane where it forms a complex with important cell signaling proteins [28]. Throughout the cytoplasm, ITSN$1 \mathrm{~s}$ is associated vesicles, cytoskeleton elements and Golgilike structures, in the perinuclear region $[8,13,26]$. Recent findings in our lab also show that ITSN-1s is present in the nucleus and interacts with important nuclear proteins (unpublished). The wide subcellular distribution of ITSN$1 \mathrm{~s}$ is consistent with its involvement of multiple important signaling pathways involved in pulmonary pathogenesis.

\section{Role of ITSN-1s in Endocytosis and cell signaling}

ITSN-1s plays an important role in endocytosis and vesicle trafficking; ITSN-1s binds to a number of important endocytic proteins and localizes to clathrin-coated pits and caveolae at the plasma membrane $[5,8,30]$. ITSN- 1 s binds several dynamin-2 molecules simultaneously and clusters them at endocytic sites creating a high concentration of dynamin-2, which is required for collar formation around the necks of endocytic vesicles, leading to membrane fission and endocytosis $[5,8,31]$. This interaction is via the $\mathrm{SH} 3$ domains of ITSN-1s [5, 8, 32]. The SH3A domain has the highest affinity for dynamin-2, and regulates its assemblydisassembly and the GTPase activity in the process of caveolae-dependent endocytosis [33]. However, ITSN-1s effects in regulating endocytosis are concentrationdependent as studies have shown that both silencing ITSN1 gene (siRNA) and overexpressing ITSN-1s inhibit

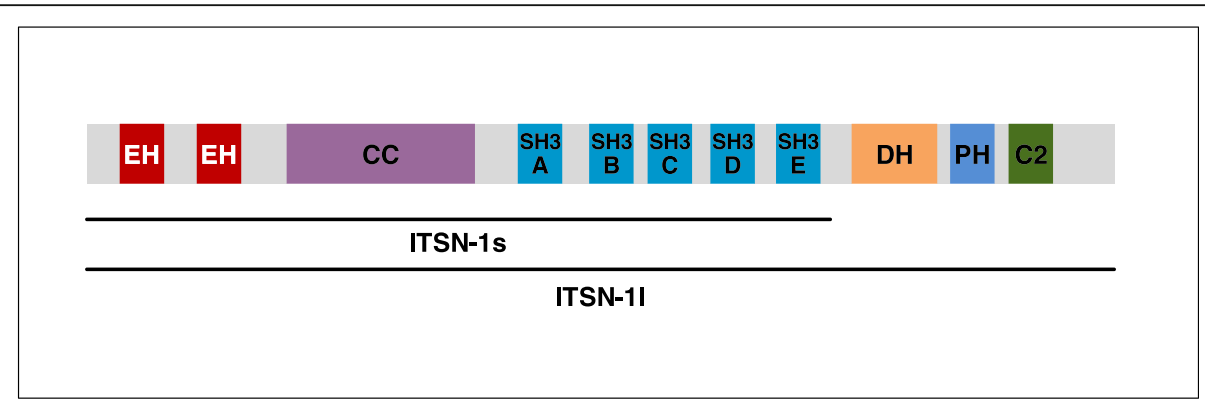

Fig. 1 The domain structure intersectin-1 (ITSN-1) proteins. ITSN-1 proteins are comprised of Eps15 homology (EH) domains, a coiled-coil (CC) region, and 5 consecutive Src homology 3 (SH3) domains (SH3A, SH3B, SH3C, SH3D, SH3E), each with distinct ligands. A longer splice variant, termed ITSN-1 I, shares all the domains with the shorter spliced isoform, referred to as ITSN-1S, but in addition possesses a C-terminal extension encoding a Dbl-homology domain $(\mathrm{DH})$, a pleckstrin homology $(\mathrm{PH})$ domain and a $\mathrm{C} 2$ domain 
endocytosis [32, 33]. Overexpression of SH3A in endothelial cells (EC) as well as in mouse lung endothelium stimulates dynamin-2 assembly and stabilizes dynamin-2 oligomers preventing detachment of caveolae from the plasma membrane and as result, acting as a potent inhibitor of endocytosis [32, 33]. Similarly, acute depletion of ITSN$1 \mathrm{~s}$ in mouse lungs results in inefficient dynamin-2 recruitment to the endocytic site leading to a decreased number of free caveolae. This impairs trans-endothelial transport which in turn opens interendothelial junctions and activates paracellular transport. Prolonged inhibition of ITSN-1s upregulates alternative endocytic structures/pathways which partially restore the junctional integrity [32].

Studies have also demonstrated that ITSN-1s regulates a number of important cell signaling pathways [1]. Highthroughput yeast two-hybrid screening identified more than 100 interacting proteins with ITSNs (55 binding proteins for ITSN-1, 62 binding proteins for ITSN-2). In addition, ITSN-1 and ITSN-2 can dimerize with itself or each other [1]. Given the wide subcellular distribution of ITSN-1s, it has unique interactions and regulates different signaling pathways depending on the intracellular compartment and its spatial orientation.

Although ITSN-1s does not have a guanine nucleotide exchange factor (GEF) domain like ITSN-1 l, [C-terminal DH-PH domains act as a GEF [34, 35]], it plays an important role in the regulation of multiple Ras family GTPases $[9,10,13]$. Ras family proteins regulate many processes in the cell, including MAPK signaling, cell proliferation, cytoskeleton organization and cell migration [36]. Ras GTPases cycle between an active GTP-bound form and an inactive GDP-bound form [37, 38]. This cycling is regulated by GEFs that promote release of GDP and subsequent binding of GTP to activate Ras. GTPase activating or accelerating proteins (GAPs) enhance the intrinsic GTPase activity to promote GTP hydrolysis to terminate Ras GTPase activity [37, 39-42]. ITSN-1s interacts with CdGAP, mSos1 and Eps8 which regulate the activity of GTPase proteins Cdc42, Ras and Rac1 respectively [6, 9, 10, 13, 24, 43]. ITSN-1s' interaction with CdGAP inhibits its GAP activity leading to activation of Cdc42 and Rac1 [24, 43]. This interaction is tissue/organ dependent; splicing of five additional amino acids in microexon 20 within the SH3A domain of ITSN-1s leads to enhanced interaction with CdGAP in brain tissue, and reduced binding in lung tissue [29].

ITSN-1s' interaction with mSos1 is more complex. The SH3 domains of ITSN-1s form a stable complex with $\mathrm{mSos} 1$ and compete with Grb2 for binding to the same site on mSos1 $[10,12]$. Activation of RTK recruits mSos1-Grb2 complex to activate Ras and MAPK pathway. Consistent with this, overexpression of the SH3 domains of ITSN alone impairs mSos1-Grb2 complex and prevents Ras-mediated MAPK activation $[9,10]$. However, overexpression of full- length ITSN-1s activates Ras in the perinuclear vesicles without downstream activation of the MAPK pathway [6]. ITSN-1s mediated Ras activation in the perinuclear vesicles was initially thought to be via mSos1, but recent studies have disproved this observation and have implicated a novel PI3K isoform in this interaction [1]. The novel PI3K isoform, PI3KC2 $\beta$, preferentially binds to inactive Ras and inhibits its activation. In the presence of ITSN-1s, the preferential interaction of PI3KC2 $\beta$ and ITSN-1s results in dissociation from inactive Ras which makes it available for immediate activation by GTP loading [44].

Eps8 interacts with mSos1 in the presence of RTK activation to convert the Rac1 GTPase from its inactive GDPbound state to the active GTP-bound state [45]. ITSN-1s impairs mSos1-Eps8 interaction and favors Cbl-Eps8 interaction leading to impaired Rac1 activation and Eps8 ubiquitination, respectively. $\mathrm{Cbl}$ is an E3 ubiquitin ligase and plays an important role in regulating the levels of numerous proteins [46]. These interactions are most likely between the SH3 domain(s) of ITSN-1s and the proline-rich regions of Eps8 and $\mathrm{Cbl}$, similar to ITSN's interactions with most signaling proteins [22]. The multiple domains of ITSN-1s are known to simultaneously interact with the same protein as well as different proteins [5]. Given the presence of five SH3 domains, ITSN-1s is able to simultaneously interact with multiple proline-rich domains of proteins to coordinate different cellular signaling processes $[7,25]$. Rac1 has a complex reciprocal relationship with RhoA, and the balance between the two determines cell migration by reorganizing cytoskeleton elements and focal adhesions [47, 48]. Consistent with this, impaired Rac1 activation in the presence of ITSN-1s leads to increased RhoA activation [13].

ITSN-1s also plays an important role in RTK's regulation of numerous signaling pathways [28]. RTK function is regulated by endocytosis and ubiquitination. Although endocytosis terminates RTK signaling, it has emerged as a prerequisite step in the activation of signaling pathways $[25,49]$. Internalization and trafficking of the epidermal growth factor receptor (EGFR) to endosomes is necessary for maximal activation of the MAPK pathway by EGFR [28]. Consistent with this, silencing ITSN-1s gene attenuates the extent and duration of Erk $1 / 2$ activation after epithelial growth factor stimulation, an effect that is due to decreased EGFR internalization [28]. ITSN-1s also decreases EGFR activity by promoting ubiquitination of EGFR by the ubiquitin E3 ligase Cbl [28]. In addition, ITSN-1s interacts with a number of other proteins involved in the regulation of $\mathrm{Cbl}$ such as sprouty2 and CIN85 [1, 50, 51].

\section{ITSN-1s deficiency in lung cancer}

ITSNs' participation in the activation of numerous mitogenic kinases provided strong suspicion for a potential involvement in cell proliferation and cancer. Earlier 
studies have shown that overexpression of ITSN-1s induces oncogenic transformation of rodent fibroblasts [52]. Both low and high levels of ITSN-1s have been reported in several cancers. The first clinical evidence for the role of ITSN-1s in cancer came from patients with Down syndrome [53]. The human ITSN1 maps proximal to the Down syndrome critical region (21q22.1-q22.2 between markers D21S319 and D21S65). Triplication (trisomy) of this region is associated with many phenotypes of Down syndrome [19]. As a consequence of ITSN-1s' location in the down syndrome critical region, these patients have an elevated ITSN-1 mRNA and protein level [54]. In a cohort prospective registry study, these patients had a significantly lower incidence of solid organ cancers, especially lung cancer, compared to the age matched general population [53]. In addition, the $\mathrm{Hu}$ man Protein Atlas reports significantly lower levels of ITSN-1s protein level in lung cancer and a number of other solid organ malignancies [55]. However, studies in neuroblastoma and glioblastoma have shown upregulation of ITSN-1s and reduced tumorigenesis and cell migration with silencing of ITSN-1s gene $[44,56,57]$. This demonstrates that ITSN-1s mediated interactions and regulation of signaling pathways is specific to the tissue and disease. The numerous minor splice variants that occur throughout the ITSN-1s protein, results in changes in the $\mathrm{EH}, \mathrm{SH} 3$, and $\mathrm{DH}$ domains and facilitate these tissue and disease-specific interactions [4]. Alternative splicing generates protein isoforms with different biological properties that differ in protein:protein interactions, subcellular localization, protein stability and posttranslational modifications [58]. ITSN isoforms have also been demonstrated to play a causative role in other diseases such as. glioblastoma, neuroblastoma, Alzheimer's disease, Down syndrome, retinitis pigmentosa and tracheomalacia $[44,56,57,59-61]$. It is increasingly recognized that the large number of alternative splicing events of ITSN-1s mRNA, the alternative polyadenylation, the tissue-specific expression level of different transcripts and their differential binding to interacting partners, play an important role in regulation of ITSN$1 \mathrm{~s}$ in health and disease [27, 62, 63]. The detailed characterization of isoform specific-functions of ITSN$1 \mathrm{~s}$, and how specific changes in splicing impact the disease process and risk require further investigation.

The level of both ITSN-1s protein and mRNA is significantly decreased in human lung cancer likely due to suppression of transcription [13]. Pilot studies using immunohistochemistry staining in lung cancer tissue samples demonstrate a negative correlation between the ITSN-1s protein level and the aggressiveness of lung cancer. The subcellular distribution of ITSN-1s is not altered in lung cancer [13]. The impact of ITSN-1s deficiency in lung cancer progression was studied by comparing control lung cancer cells (A549) to those with ITSN-1s protein level restored to normal levels by stable transfection using a myc-ITSN-1s plasmid (A549 + ITSN-1s). ITSN-1s expression impairs lung cancer cell proliferation, anchorage-independent growth and tumorigenesis [13]. We have shown that ITSN-1s expression in lung cancer cells impairs Eps8 interaction with mSos1 and facilitates Eps8-Cbl interaction leading to ubiquitination and downregulation of Eps8. Eps8 interacts with mSos 1 in the presence of RTK activation to convert the Rac1 GTPase from its inactive GDP-bound state to the active GTP-bound state [45]. Impaired Eps8mSos1 interaction in the presence of ITSN-1s decreases Rac1 activation. Rac1 activation is a required for Rasmediated tumor progression [64]. Rac1 activation also directly activates the JNK pathway leading to tumor progression [65]. As an oncoprotein, Eps8 also translocates to the nucleus and upregulates numerous cell cycle proteins such as transcription factor Foxm1 [66]. Therefore, ITSN-1s mediated inhibition of tumorigenesis is likely due to a combined inhibitory effect on these pathways. In addition, ITSN-1s enhances ubiquitination and downregulation of EGFR with a potential negative impact on proliferation [28].

In the presence of ITSN-1s, decreased Rac1 activation and reciprocal upregulation of RhoA shift the balance in favor of decreased lung cancer cell migration and metastasis [13]. This results in a number of significant changes in the cytoskeleton. In the presence of ITSN-1s, lung cancer cells show phenotypic changes favoring transition from mesenchymal-to-epithelial cells: increased spreading, lack of elongated and polarized morphology, and prominent actin bundles towards peripheral attachment points [13]. In addition, the number of vinculin focal adhesions at the cell surface is increased and the vimentin filament network is collapsed. In a scratch closure assay (in vitro cell migration model) ITSN-1 mediated cytoskeleton changes lead to significantly decreased cell migration and scratch closure. In vivo mouse metastasis assay shows significantly more tumor metastasis to the lungs as well as larger size tumors in A549 cancer cells compared to A549 + ITSN-1s cells. Altogether, these studies demonstrate that ITSN-1s deficiency is a crucial event in lung cancer progression. ITSN-1s' ability to reverse the malignant features demonstrates the capability of this protein to regulate multiple pathways simultaneously which makes it an attractive therapeutic target. Further validation of ITSN-1s protein level in a large cohort of patients at different stages of lung cancer could establish ITSN-1s as a predictor of prognosis and indicator of response to therapy. Given the ubiquitous distribution of ITSN-1s and evidence that loss of ITSN-1s is a characteristic feature of many cancers [55], these findings may be applicable to other types of cancer. 
ITSN-1s deficiency in acute lung injury

Acute lung injury is associated with excessive apoptosis of pulmonary endothelial and epithelial cells, which induce endothelial and epithelial barrier dysfunction leading to pulmonary edema $[67,68]$. Acute lung injury is triggered by direct injury to the lung and can be secondary to other inflammatory conditions such as sepsis, pancreatitis or transfusion rejection. Studies have shown that apoptotic pulmonary cells or macrophages engulfing apoptotic cells release substances such as growth factors, and create conditions that favor the emergence of apoptosis-resistant cells [68, 69].

In acute lung injury, as in many pro-inflammatory states, full-length ITSN-1s expression is decreased [11, 12, 68]. ITSN-1s is important for the pro-survival signaling pathway mSos1/Ras/Erk1/2 MAPK which regulates cell survival, proliferation and vascular remodeling $[6,9,10]$. Downregulation of ITSN-1s, via siRNA, inhibits the Erk1/2 MAPK pathway leading to mitochondrial apoptosis of EC [30]. However, EC with LPS-induced ITSN-1s deficiency do not undergo apoptosis since ITSN-1s deficiency is countered by upregulation of anti-apoptotic proteins leading to protection of EC [16].

The use of ITSN-1s knockdown (KD $\left.\mathrm{K}_{\text {ITSN }}\right)$ mouse model has significantly advanced our understanding of the mechanisms involved in lung injury as well as the role of ITSN$1 \mathrm{~s}$ in the pathogenesis of acute lung injury. $\mathrm{KD}_{\text {ITSN }}$ mouse model was generated by intravenous delivery of the cat-

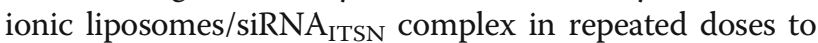
maintain continuous downregulation of ITSN-1s protein expression up to 24 days. The amount injected $(100 \mu \mathrm{g}$ siRNA/mouse) was efficient and specific in inhibiting ITSN-1s mRNA and protein levels without significant adverse effects [11].

Acute $\mathrm{KD}_{\text {ITSN }}$ (assessed $72 \mathrm{~h}$ post-siRNA delivery) leads to decreased Erk1/2 MAPK signaling and causes significant EC apoptosis, microvascular loss and alveolar destruction. The lung injury is patchy and mostly involves the alveolar capillary units centered on small vessels and mid-sized vessels [11]. The morphology of ECs nuclei, mitochondria, and Golgi are affected as well. As a consequence, the alveolar-capillary unit becomes increasingly permeable leading to leakage of protein-rich fluid from the vascular to the interstitial space causing distension of the interstitial space [11]. These findings are identical to the pathophysiological processes that are hallmarks of acute lung injury [68]. This pattern of lung injury triggered by ITSN-1s deficiency persists up to about 10 days.

ITSN-1s interaction with endocytic proteins is crucial for normal endocytosis [8]. The pulmonary EC of $\mathrm{KD}_{\text {ITSN }}$ mice demonstrate impaired normal endocytosis which occurs via caveolin and clathrin-coated vesicles [12]. ITSN-1s deficiency leads to upregulation of alternative endocytic pathways (ECs display enlarged endocytic structures, membranous rings and tubules) to compensate for deficiency in normal endocytosis and vesicular [32]. Both endocytosis and vesicular trafficking are crucial for growth factor receptor signaling and activity of their downstream targets [70]. TGF $\beta$ is a ubiquitous and multifunctional cytokine which in the setting of lung injury expresses antiinflammatory properties confined to the extent of septal injury and facilitates recovery [71]. Alk5 is a widely expressed transforming growth factor $\beta$ receptor I (TGF $\beta$ RI) which complexes with TGF $\beta$ RII when activated by growth factor TGF $\beta$, and activates Smad2 and Smad3 proteins via the canonical TGF $\beta$ signaling pathway [72, 73]. In normal EC, internalization of Alk5 via clathrin-coated vesicles leads to TGF $\beta$-induced activation of $\operatorname{Smad} 2 / 3$ pathway which is then recycled to the plasma membrane. In contrast internalization of Alk5 by caveolae is directed to ubiquitin proteasome pathway [73, 74]. The alternative pathways upregulated by ITSN-1s deficiency express predominantly caveolin-1 and therefore alters the endocytic trafficking of Alk5 in favor of enhanced degradation [12]. Consistent with this, in acute lung injury Alk5 expression is significantly decreased up to 10 days; however after 10 days Alk5 expression starts to gradually recover and reaches normal levels by 24 days [11]. The recovery of Alk 5 expression is via the effects of microparticles. Microparticles are 0.5-1.0 $\mu \mathrm{m}$ diameter in size and play a crucial role in the communication between different cell types in normal and pathological settings. They have double membrane morphology and store important bio-effectors which play a crucial role in the recovery of lung injury by inducing endothelial modifications via membrane fusion and paracrine effects $[68,75]$. At day 10 of ITSN-1s deficiency when EC apoptosis and lung injury are at their peak, there is a significant increase in microparticles containing Alk5. In the setting of prolonged ITSN-1s deficiency these microparticles are able to interact and transfer Alk5/TGF $\beta$ RII complexes to dysfunctional EC [11]. The expression of TGF $\beta$ is also significantly increased at 10 days when lung injury is at its peak. As a result of increased TGF $\beta$ and restoration of its receptor Alk5/ TGFßRI, the Erk1/2 MAPK pathway is restored, and remaining non-apoptotic quiescent EC exhibit phenotypic changes toward hyperproliferation and apoptosis resistance leading to increased microvessel density, repair and remodeling of the lungs [11]. Within 2 weeks after severe injury, mouse lung function returned to normal state with little evidence of prior damage. A lung repair process characterized by EC proliferation and increased microvascular density was critical for the remarkable recovery. However, the typical TGF $\beta /$ ALK5 signaling is shifted from Smad2/3 activation towards a less common Ras/Erk1/2 MAPK pathway [12]. Since ITSN-1s associates with mSos1 in a complex that excludes Grb2, ITSN-1s deficiency increases mSos1 availability for Grb2 interaction and results in preferential formation of ALK5/mSos1/Grb2 signaling complex. This 
shifts the balance from Smad2/3-Erk1/2 towards Ras/ MEK/Erk1/2 activation [12]. Despite continuous and efficient $\mathrm{KD}_{\mathrm{ITSN}}$, apoptosis of EC starts to decrease after 10 days, and by 24 days has reached almost normal levels. A similar proliferative pattern was also seen with epithelial alveolar cells.

As the timeline of acute lung injury/acute respiratory distress syndrome (ARDS) related pathology is shared by both mice and humans, and since ITSN-1s deficiency is also a characteristic of human lung tissue of acute lung injury/ARDS patients [76], we applied a translational approach to study microparticles from the blood of ARDS patients. Similar to the mouse studies, we have identified a population of Alk5/TGF $\beta$ RI-immunoreactive microparticles [76]. Flow cytometry and calibrated/counted beads used to quantify the microparticles indicated that the Alk5-positive microparticle population is more numerous in the ARDS patients compared to healthy controls, consistent with reports of elevated levels of microparticles in disease settings [77]. Flow cytometry and magnetic bead separation via biotin-conjugated Alk5 Ab and streptavidin magnetic beads demonstrated that these Alk5-positive microparticles are immunoreactive to CD73 and CD105 and negative to CD34 CD45, suggesting a mesenchymal stem cell origin [78]. Additional studies demonstrate that these particles interact with LPS-treated lung ECs, in culture and in vivo, leading to improved permeability and decrease in lung histological severity, consistent with longterm follow-up studies of ARDS survivors, suggesting that lung repair is in fact a hallmark of the normal course of recovery from acute lung injury [79]. Altogether, these observations demonstrate that extensive pulmonary EC death due to ITSN-1s deficiency stimulates mesenchymal stem cell paracrine signaling via microparticles leading to generation of hyperproliferative and apoptotic-resistant endothelial cells and subsequent recovery of lung injury.

\section{ITSN-1s deficiency in pulmonary arterial hypertension}

Pulmonary arterial hypertension (PAH) is a disease in which there is persistent elevation of pulmonary artery pressure. The pathological findings in this disease are medial hypertrophy, intimal proliferation and fibrosis of pulmonary arteries and arterioles [80]. The intimal changes lead to progressive obstruction of the vessels which, in the presence of high pressure, dilate and evolve into microaneurysms. Areas of microaneurysms lead to endothelial proliferation and formation of in situ thrombosis which leads to the characteristic plexiform lesions. The obliteration of vessels and plexiform lesions lead to an increase in pulmonary vascular resistance and ultimately right heart failure. Enhanced proliferation of EC, smooth muscle cells and fibroblasts are central to the pathogenesis of PAH [81]. Emergence of proliferative $\mathrm{ECs}$ in $\mathrm{PAH}$ is a consequence of initial EC dysfunction and apoptosis and subsequent selection of apoptoticresistant proliferative EC [82].

Inflammatory mechanisms play a significant role in the initiation of the pathogenesis of PAH. Inflammation associated with PAH attracts inflammatory cells to release granzyme B [83]. Interestingly, ITSN-1s is a substrate for granzyme $B$ with a cleavage site at the IDQD ${ }^{271 G K}$ sequence, a well-conserved sequence among mammals [84]. The cleavage results in decreased expression of full-length ITSN-1s protein and in two biologically active protein fragments, N-terminal fragment $\left(\mathrm{EH}_{\mathrm{ITSN}}\right)$ and C-terminal fragment (SH3A-E $\mathrm{E}_{\mathrm{ITSN}}$ ) [15]. Evidence demonstrates that ITSN-1s is a substrate for granzyme B and its cleavage leading to deficiency of ITSN-1s which is associated with the pathogenesis of PAH [15]. Mice treated with lipopolysaccharide (LPS; bacterial endotoxin which induces a strong immune response and leads to increase in granzyme B) and monocrotaline-induced PAH mouse and rat models results in loss of full-length ITSN-1s expression and the presence of a $28-\mathrm{kDa}$ fragment corresponding to the molecular weight of $\mathrm{EH}_{\text {ITSN }}$ [15]. Biochemical analyses of lung ECs of PAH patients demonstrate low levels of full-length ITSN-1s protein and mRNA expression. Immunohistochemistry staining of human PAH specimens show lower ITSN-1s staining of pulmonary arteries with proliferative ECs and plexiform lesions whereas the presence of granzyme B is increased in the milieu of these lesions [15].

We have reported that both fragments formed by cleavage of full-length ITSN-1s are biologically active and impact EC proliferation $[14,15]$. $\mathrm{EH}_{\mathrm{ITSN}}$ enhances EC proliferation via activation of p38 MAPK pathway. In the presence of increased $\mathrm{EH}_{\mathrm{ITSN}}$ expression, p38 MAPK is distributed predominantly in the cytosol and is activated. P38 MAPK activation leads to activation of Elk-1 transcription factor which facilitates Elk-1 binding to the c-Fos promoter leading to increased expression of the growth related protein cFos. Treatment with a selective p38 MAPK inhibitor significantly inhibits EC proliferation confirming the role of $\mathrm{EH}_{\text {ITSN }}$ mediated activation of p38 MAPK in EC proliferation. $\mathrm{EH}_{\mathrm{ITSN}}$ has no effect on JNK or the PI3K/Akt signaling pathway, and impairs Erk1/2 MAPK activation via negative cross talk from p38 to Erk1/2. The SH3A- $\mathrm{E}_{\text {ITSN }}$ fragment, however, impairs proliferation by sequestering mSos1 and inhibiting activation of Ras/Erk1/2 MAPK signaling. The concurrent expression of both $\mathrm{EH}_{\mathrm{ITSN}}$ and SH3A- $\mathrm{E}_{\text {ITSN }}$ results in a high $\mathrm{p} 38 /$ Erk1/2 MAPK activity ratio favoring EC proliferation [15].

ITSN-deficient mice transduced with $\mathrm{EH}_{\mathrm{ITSN}}$ developed pathological findings similar to $\mathrm{PAH}$ patients. Wild-type mice and ITSN-deficient mice $\left[\mathrm{KD}_{\mathrm{ITSN}}\right.$ and ITSN knockout/heterozygous $\left(\mathrm{KO}_{\mathrm{ITSN}+/-}\right)$ ] were treated with myc$\mathrm{EH}_{\mathrm{ITSN}}$ cationic lipoplexes delivered repeatedly by retroorbital injection every $48 \mathrm{~h}$ for 20 days. No hypoxia or chemical/synthetic compounds known to induce PAH were 
used. $\mathrm{EH}_{\mathrm{ITSN}}$ transduced ITSN-deficient mice developed numerous and widespread clusters of proliferating pulmonary ECs and pathological findings consistent with plexiform lesions; hypercellular and stalk-like lesions arising from the vessel walls protruded into the lumen of the pulmonary artery and caused severe obliteration of the vessel. These lesions displayed a rich matrix of collagen consistent with vascular medial thickening seen in human PAH [85]. Moreover, proliferation of pulmonary artery smooth muscle cells was also observed in the lung vessels of $\mathrm{EH}_{\mathrm{ITSN}}$-transduced ITSN-1s deficient mice, suggesting significant cross-talk between ECs and smooth muscle cells leading to the pathogenesis of $\mathrm{PAH}$ which involves proliferation and hypertrophy of both intimal and medial layers. Only 20 days of $\mathrm{EH}_{\text {ITSN }}$ treatment, independent of any other insults, resulted in modest increase in the RVSP values (from 21 to $25.5 \mathrm{mmHg}$ ) and right ventricular hypertrophy.

Similar to the findings in cultured ECs, the lungs of $\mathrm{EH}_{\mathrm{ITSN}}$-transduced ITSN-deficient mice showed increased activation of p38 MAPK, Elk-1 transcription factor and increased expression of the c-Fos gene, consistent with activation of p38 MAPK pathway [14]. Prior studies have also implicated p38 MAPK in cell proliferation and vascular obliteration leading to the pathogenesis of PAH [86]. Given that the motif NPF is an essential target of EH domains [87], the proliferative potential of ECs expressing $\mathrm{EH}_{\text {ITSN }}$ is impaired by treatment with a membrane permeable peptide containing the NPF motif [88]. As $\mathrm{EH}_{\text {ITSN }}$ is a highly specific molecular target, this could be a very effective treatment option to ameliorate and perhaps reverse the EC plexiform phenotype already established in severe human PAH.

\section{Conclusion}

ITSN-1s is a multi-domain protein with numerous binding partners capable of regulating many important signaling pathways. Studies to date implicate ITSN-1s deficiency in the pathogenesis of several pulmonary diseases (Fig. 2). In lung cancer, ITSN-1s deficiency shifts the balance in favor of greater Eps8-Sos1 interaction and less Eps8-Cbl interaction leading to activation of Rac1 and increased expression of Eps8 respectively. As a result of this and other potential interactions, ITSN-1s plays a crucial role in lung cancer development and progression: proliferation, anchorage-independent growth, cytoskeleton modification, migration and metastasis. As a pro-survival protein, ITSN1 s deficiency is a crucial early event in development of acute lung injury. ITSN-1s deficiency impairs normal endothelial structures and their function which leads to endocytosis of Alk5 receptors via alternative endocytic pathways resulting in Alk5 ubiquitination and degradation. Downregulation of Alk5 expression in acute lung injury is counterbalanced by circulating microparticles which, via paracrine effects, interact and transfer Alk5/TGFßRII complex to

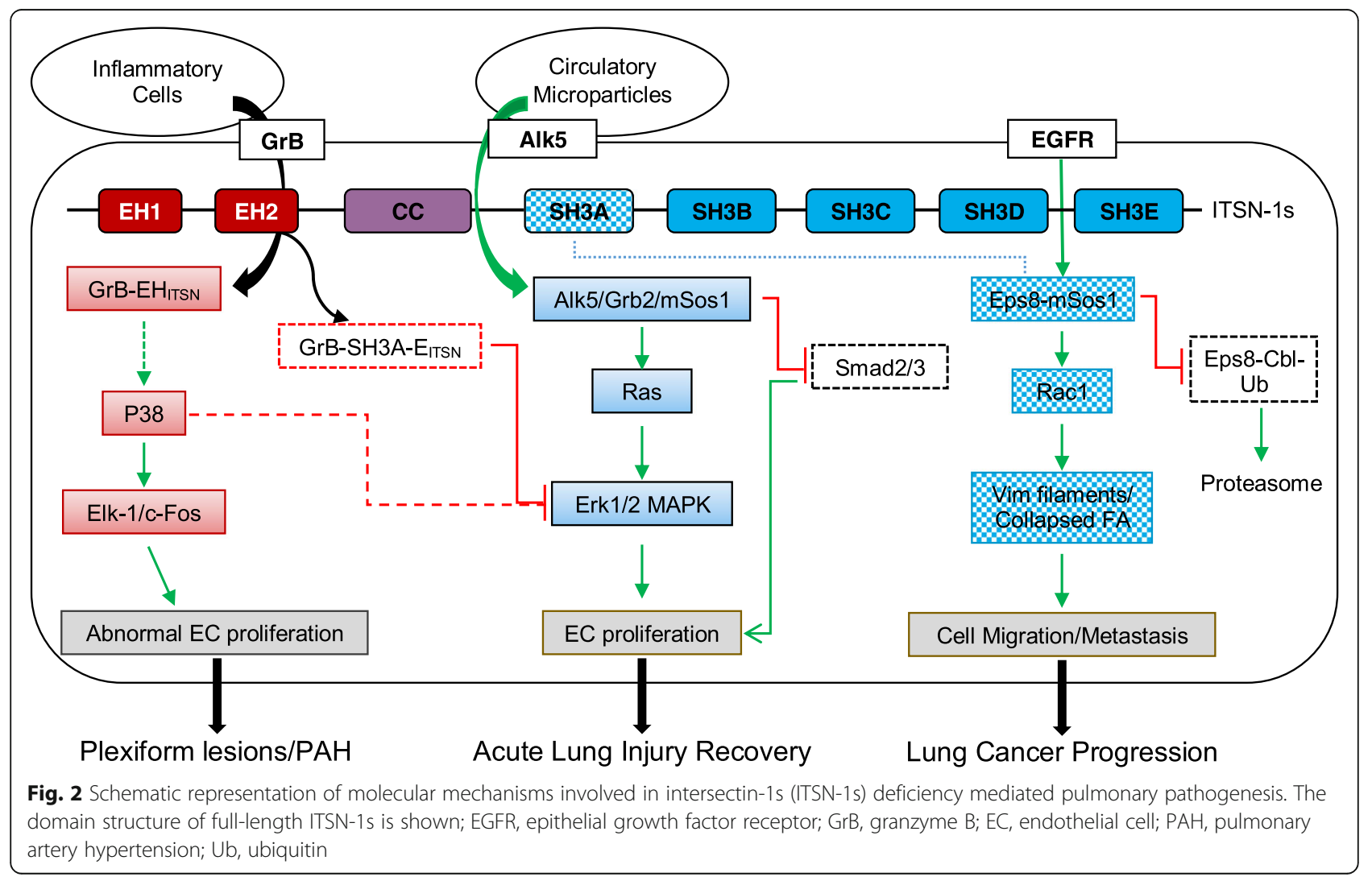


remaining non-apoptotic cells. These receptors, in a cellular context characterized by ITSN-1s deficiency and aberrant endocytosis, signal via Erk1/2 MAPK pathway, instead of the usual Smad 2/3 pathway, regardless leading to restoration and repair of lung architecture. In the setting of PAH, full-length ITSN-1s is cleaved by granzyme B released by inflammatory cells. The cleavage results in $\mathrm{EH}_{\mathrm{ITSN}}$ and SH3A- $\mathrm{E}_{\text {ITSN }}$ fragments. The $\mathrm{EH}_{\text {ITSN }}$ fragment leads to EC proliferation via activation of p38 MAPK and Elk-1/c-Fos signaling. The SH3A- $\mathrm{E}_{\text {ITSN }}$ fragment impairs Ras/Erk1/2 MAPK signaling. However, the concurrent expression of both fragments results in high p38/Erk1/2 MAPK activity favoring pulmonary cell proliferation. In vivo, ITSN-1s deficient mouse transduced with $\mathrm{EH}_{\text {ITSN }}$ plasmid leads to EC and smooth muscle proliferation resulting in pulmonary vascular obliteration and plexiform lesions consistent with pathological findings seen in severe PAH. As shown in these studies related to pulmonary diseases and others, ITSN-1s regulates multiple signaling pathways simultaneously in a tissue, concentration and subcellular distribution specific manner. Given the numerous protein-protein interactions between the multiple domains of ITSN-1s, it is highly likely that additional regulatory pathways remain to be identified. Further studies will shed light into novel mechanisms of regulation of protein, genetics and epigenetics by ITSN-1s in pulmonary diseases. Future studies should also explore the role of other ITSN transcripts and potential compensatory role in pulmonary pathogenesis. Studies of ITSN-1s involvement in acute lung injury and PAH are limited and its role in the pathophysiology of these diseases is less established. The $\mathrm{KD}_{\mathrm{ITSN}}$ and $\mathrm{KO}_{\mathrm{ITSN}+/-}$ mouse models of lung injury as well as the $\mathrm{EH}_{\mathrm{ITSN}}$-transduced $\mathrm{K}_{\mathrm{ITSN}+\text { /- }}$ mouse model of plexogenic PAH that recapitulate many of the pathological events associated with the human disease are valuable tools to further advance our understanding of ITSN-1s involvement in pulmonary pathology, and provide novel targets for treating these severe human conditions.

\section{Abbreviations}

ARDS: Acute respiratory distress syndrome; DH: Dbl homology;

EC: Endothelial cells; EGFR: Epidermal growth factor receptor; EH: Eps 15 homology; $\mathrm{EH}_{\text {ITSN }}$ and SH3A-E ITSN: N-terminal fragment and C-terminal fragment; GAPS: GTPase activating or accelerating proteins; GEF: Guanine nucleotide exchange factor; ITSN-1s: Intersectin-1s; ITSNs: Intersectins; K0 ITSN +/-: ITSN knockout/heterozygous; KD ITSN: ITSN-1s knockdown;

NPF: Asparagine-proline-phenylalanine; PAH: Pulmonary arterial hypertension; $\mathrm{PH}$ : a Pleckstrin; SH3: Src homology 3

\section{Acknowledgements}

None.

\section{Funding}

Supported by Rush Executive Committee of Research Grant (to NJ) and R01HL089462 (to SP).

Availability of data and materials

Not applicable as no data were generated or analyzed.

\section{Authors' contributions}

$\mathrm{NJ}$ drafted the article and prepared for submission. DP and SP edited the final article. All authors approved the final version of the manuscript.

Ethics approval and consent to participate

Not applicable.

\section{Consent for publication}

Not applicable.

Competing interests

The authors declare that they have no competing interest.

\section{Publisher's Note}

Springer Nature remains neutral with regard to jurisdictional claims in published maps and institutional affiliations.

\section{Author details}

${ }^{1}$ Rush University Medical Center, Chicago, IL 60612, USA. ${ }^{2}$ Department of Pharmacology and Division of Pulmonary and Critical Care Medicine, Rush University, 1750 W. Harrison Street, 1415 Jelke, Chicago, IL 60612, USA.

${ }^{3}$ Department of Pharmacology and Division of Pulmonary and Critical Care Medicine, Rush University Medical Center and Rush Medical College, 1750 W. Harrison Street, 1535 Jelke, Chicago, IL 60612, USA.

Received: 13 March 2017 Accepted: 28 August 2017

Published online: 06 September 2017

\section{References}

1. Wong KA, Wilson J, Russo A, Wang L, Okur MN, Wang X, Martin NP, Scappini E, Carnegie GK, O'Bryan JP. Intersectin (ITSN) family of scaffolds function as molecular hubs in protein interaction networks. PLoS One. 2012;7:e36023.

2. Flynn DC. Adaptor proteins. Oncogene. 2001;20:6270-2.

3. Yee D. Adaptor proteins as targets for cancer prevention. Cancer Prev Res (Phila). 2010;3:263-5.

4. Tsyba L, Nikolaienko O, Dergai O, Dergai M, Novokhatska O, Skrypkina I, Rynditch A. Intersectin multidomain adaptor proteins: regulation of functional diversity. Gene. 2011;473:67-75.

5. Okamoto M, Schoch S, Sudhof TC. EHSH1/intersectin, a protein that contains $\mathrm{EH}$ and $\mathrm{SH} 3$ domains and binds to dynamin and SNAP-25. A protein connection between exocytosis and endocytosis? J Biol Chem. 1999;274:18446-54.

6. Mohney RP, Das M, Bivona TG, Hanes R, Adams AG, Philips MR, O'Bryan JP. Intersectin activates Ras but stimulates transcription through an independent pathway involving JNK. J Biol Chem. 2003;278:47038-45.

7. O'Bryan JP. Intersecting pathways in cell biology. Sci Signal. 2010;3:re10.

8. Predescu SA, Predescu DN, Timblin BK, Stan RV, Malik AB. Intersectin regulates fission and internalization of caveolae in endothelial cells. Mol Biol Cell. 2003;14:4997-5010.

9. Tong XK, Hussain NK, Adams AG, O'Bryan JP, McPherson PS. Intersectin can regulate the Ras/MAP kinase pathway independent of its role in endocytosis. J Biol Chem. 2000;275:29894-9.

10. Tong XK, Hussain NK, de Heuvel E, Kurakin A, Abi-Jaoude E, Quinn CC, Olson MF, Marais R, Baranes D, Kay BK, McPherson PS. The endocytic protein intersectin is a major binding partner for the Ras exchange factor mSos 1 in rat brain. EMBO J. 2000;19:1263-71.

11. Bardita C, Predescu D, Justice MJ, Petrache I, Predescu S. In vivo knockdown of intersectin-1s alters endothelial cell phenotype and causes microvascular remodeling in the mouse lungs. Apoptosis. 2013;18:57-76.

12. Bardita C, Predescu DN, Sha F, Patel M, Balaji G, Predescu SA. Endocytic deficiency induced by ITSN-1s knockdown alters the Smad2/3-Erk1/2 signaling balance downstream of Alk5. J Cell Sci. 2015;128:1528-41.

13. Jeganathan N, Predescu D, Zhang J, Sha F, Bardita C, Patel M, Wood S, Borgia JA, Balk RA, Predescu S. Rac1-mediated cytoskeleton rearrangements induced by intersectin-1s deficiency promotes lung cancer cell proliferation, migration and metastasis. Mol Cancer. 2016;15:59.

14. Patel M, Predescu D, Bardita C, Chen J, Jeganathan N, Pritchard M, DiBartolo S, Machado R, Predescu S. Modulation of Intersectin-1s Lung Expression Induces Obliterative Remodeling and Severe Plexiform Arteriopathy in the Murine Pulmonary Vascular Bed. Am J Pathol. 2017;187(3):528-42. 
15. Patel M, Predescu D, Tandon R, Bardita C, Pogoriler J, Bhorade S, Wang M, Comhair S, Hemnes AR, Chen J, et al. A novel p38 mitogen-activated protein kinase/elk-1 transcription factor-dependent molecular mechanism underlying abnormal endothelial cell proliferation in plexogenic pulmonary arterial hypertension. J Biol Chem. 2013;288:25701-16.

16. Singla S, Predescu D, Bardita C, Wang M, Zhang J, Balk RA, Predescu S. Proinflammatory endothelial cell dysfunction is associated with intersectin-1s down-regulation. Respir Res. 2011;12:46.

17. Yamabhai M, Hoffman NG, Hardison NL, McPherson PS, Castagnoli L, Cesareni G, Kay BK. Intersectin, a novel adaptor protein with two Eps15 homology and five Src homology 3 domains. J Biol Chem. 1998;273:31401-7.

18. Kelly LE, Phillips AM. Molecular and genetic characterization of the interactions between the drosophila stoned-B protein and DAP-160 (intersectin). Biochem J. 2005;388:195-204.

19. Guipponi M, Scott HS, Chen H, Schebesta A, Rossier C, Antonarakis SE. Two isoforms of a human intersectin (ITSN) protein are produced by brainspecific alternative splicing in a stop codon. Genomics. 1998;53:369-76.

20. Hunter MP, Russo A, O'Bryan JP. Emerging roles for Intersectin (ITSN) in regulating signaling and disease pathways. Int J Mol Sci. 2013;14:7829-52.

21. Pucharcos C, Casas C, Nadal M, Estivill X, de la Luna S. The human intersectin genes and their spliced variants are differentially expressed. Biochim Biophys Acta. 2001;1521:1-11

22. Novokhatska O, Dergai M, Tsyba L, Skrypkina I, Filonenko V, Moreau J, Rynditch A. Adaptor proteins intersectin 1 and 2 bind similar proline-rich ligands but are differentially recognized by $\mathrm{SH} 2$ domain-containing proteins. PLoS One. 2013;8:e70546.

23. Pucharcos C, Estivill X, de la Luna S. Intersectin 2, a new multimodular protein involved in clathrin-mediated endocytosis. FEBS Lett. 2000;478:43-51.

24. Jenna S, Hussain NK, Danek El, Triki I, Wasiak S, McPherson PS, LamarcheVane $\mathrm{N}$. The activity of the GTPase-activating protein CdGAP is regulated by the endocytic protein intersectin. J Biol Chem. 2002;277:6366-73.

25. O'Bryan JP, Mohney RP, Oldham CE. Mitogenesis and endocytosis: What's at the INTERSECTIoN? Oncogene. 2001;20:6300-8.

26. Hussain NK, Yamabhai M, Ramjaun AR, Guy AM, Baranes D, O'Bryan JP, Der CI, Kay BK, McPherson PS. Splice variants of intersectin are components of the endocytic machinery in neurons and nonneuronal cells. J Biol Chem. 1999;274:15671-7.

27. Kropyvko S, Gerasymchuk D, Skrypkina I, Dergai M, Dergai O, Nikolaienko O, Rynditch A, Tsyba L. Structural diversity and differential expression of novel human intersectin 1 isoforms. Mol Biol Rep. 2010;37:2789-96.

28. Martin NP, Mohney RP, Dunn S, Das M, Scappini E, O'Bryan JP. Intersectin regulates epidermal growth factor receptor endocytosis, ubiquitylation, and signaling. Mol Pharmacol. 2006;70:1643-53.

29. Tsyba L, Gryaznova T, Dergai O, Dergai M, Skrypkina I, Kropyvko S, Boldyryev O, Nikolaienko O, Novokhatska O, Rynditch A. Alternative splicing affecting the SH3A domain controls the binding properties of intersectin 1 in neurons. Biochem Biophys Res Commun. 2008;372:929-34.

30. Predescu SA, Predescu DN, Knezevic I, Klein IK, Malik AB. Intersectin-1S regulates the mitochondrial apoptotic pathway in endothelial cells. J Biol Chem. 2007;282:17166-78.

31. Oh P, Mclntosh DP, Schnitzer JE. Dynamin at the neck of caveolae mediates their budding to form transport vesicles by GTP-driven fission from the plasma membrane of endothelium. J Cell Biol. 1998;141:101-14.

32. Predescu DN, Neamu R, Bardita C, Wang M, Predescu SA. Impaired caveolae function and upregulation of alternative endocytic pathways induced by experimental modulation of intersectin-1s expression in mouse lung endothelium. Biochem Res Int. 2012;2012:672705.

33. Knezevic I, Predescu D, Bardita C, Wang M, Sharma T, Keith B, Neamu R, Malik $A B$, Predescu S. Regulation of dynamin-2 assembly-disassembly and function through the SH3A domain of intersectin-1s. J Cell Mol Med. 2011;15:2364-76.

34. Hussain NK, Jenna S, Glogauer M, Quinn CC, Wasiak S, Guipponi M, Antonarakis SE, Kay BK, Stossel TP, Lamarche-Vane N, McPherson PS. Endocytic protein intersectin-I regulates actin assembly via Cdc42 and N-WASP. Nat Cell Biol. 2001;3:927-32.

35. Zamanian $\mathrm{JL}$, Kelly RB. Intersectin $1 \mathrm{~L}$ guanine nucleotide exchange activity is regulated by adjacent src homology 3 domains that are also involved in endocytosis. Mol Biol Cell. 2003;14:1624-37.

36. Simanshu DK, Nissley DV, McCormick F. RAS proteins and their regulators in human disease. Cell. 2017;170:17-33.

37. Hwang HS, Hwang SG, Yoon KW, Yoon JH, Roh KH, Choi EJ. CllA negatively regulates the Ras-Erk1/2 signaling pathway through inhibiting the Rasspecific GEF activity of SOS1. J Cell Sci. 2014;127:1640-6.
38. Pylayeva-Gupta Y, Grabocka E, Bar-Sagi D. RAS oncogenes: weaving a tumorigenic web. Nat Rev Cancer. 2011;11:761-74.

39. Heasman SJ, Ridley AJ. Mammalian rho GTPases: new insights into their functions from in vivo studies. Nat Rev Mol Cell Biol. 2008;9:690-701.

40. Hwang HS, Hwang SG, Cho JH, Chae JS, Yoon KW, Cho SG, Choi EJ. CIIA functions as a molecular switch for the Rac1-specific GEF activity of SOS1. J Cell Biol. 2011;195:377-86.

41. Innocenti M, Frittoli E, Ponzanelli I, Falck JR, Brachmann SM, Di Fiore PP, Scita G. Phosphoinositide 3-kinase activates Rac by entering in a complex with Eps8, Abi1, and Sos-1. J Cell Biol. 2003;160:17-23.

42. Innocenti M, Tenca P, Frittoli E, Faretta M, Tocchetti A, Di Fiore PP, Scita G. Mechanisms through which Sos-1 coordinates the activation of Ras and Rac. J Cell Biol. 2002;156:125-36.

43. Primeau M, Ben Djoudi Ouadda A, Lamarche-Vane N. Cdc42 GTPaseactivating protein (CdGAP) interacts with the SH3D domain of Intersectin through a novel basic-rich motif. FEBS Lett. 2011;585:847-53.

44. Russo A, O'Bryan JP. Intersectin 1 is required for neuroblastoma tumorigenesis. Oncogene. 2012;31:4828-34.

45. Scita G, Tenca P, Areces LB, Tocchetti A, Frittoli E, Giardina G, Ponzanelli I, Sini P, Innocenti M, Di Fiore PP. An effector region in Eps8 is responsible for the activation of the Rac-specific GEF activity of Sos-1 and for the proper localization of the Rac-based actin-polymerizing machine. J Cell Biol. 2001;154:1031-44.

46. Schmidt $\mathrm{MH}$, Dikic I. The Cbl interactome and its functions. Nat Rev Mol Cell Biol. 2005;6:907-18.

47. Parri M, Chiarugi P. Rac and rho GTPases in cancer cell motility control. Cell Commun Signal. 2010;8:23

48. Sander EE, ten Klooster JP, van Delft S, van der Kammen RA, Collard JG. Rac downregulates rho activity: reciprocal balance between both GTPases determines cellular morphology and migratory behavior. J Cell Biol. 1999; 147:1009-22.

49. McPherson PS, Kay BK, Hussain NK. Signaling on the endocytic pathway. Traffic. 2001;2:375-84

50. Okur MN, Ooi J, Fong CW, Martinez N, Garcia-Dominguez C, Rojas JM, Guy G, O'Bryan JP. Intersectin 1 enhances Cbl ubiquitylation of epidermal growth factor receptor through regulation of Sprouty2-Cbl interaction. Mol Cell Biol. 2012;32:817-25.

51. Okur MN, Russo A, O'Bryan JP. Receptor tyrosine kinase ubiquitylation involves the dynamic regulation of Cbl-Spry2 by intersectin 1 and the Shp2 tyrosine phosphatase. Mol Cell Biol. 2014;34:271-9.

52. Wang JB, Wu WJ, Cerione RA. Cdc42 and Ras cooperate to mediate cellular transformation by intersectin-L. J Biol Chem. 2005;280:22883-91.

53. Patja K, Pukkala E, Sund R, livanainen M, Kaski M. Cancer incidence of persons with down syndrome in Finland: a population-based study. Int J Cancer. 2006:118:1769-72.

54. Liu C, Belichenko PV, Zhang L, Fu D, Kleschevnikov AM, Baldini A, Antonarakis SE, Mobley WC, Yu YE. Mouse models for down syndromeassociated developmental cognitive disabilities. Dev Neurosci. 2011;33: 404-13.

55. The Human Protein Atlas [http://www.proteinatlas.org/ENSG00000205726-ITSN1/ pathology/]. Accessed 20 Aug 2017.

56. Gu F, Zhang H, Qin F, Liu X, Li W, Fu L, Ying G, Li B, Zhang M, Ma Y. Intersectin1-s, A multidomain adapter protein, Is essential for malignant glioma proliferation. Glia. 2015;63(9):1595-605.

57. Ma Y, Wang B, Li W, Ying G, Fu L, Niu R, Gu F. Reduction of intersectin1-s induced apoptosis of human glioblastoma cells. Brain Res. 2010;1351:222-8.

58. Stamm S, Ben-Ari S, Rafalska I, Tang Y, Zhang Z, Toiber D, Thanaraj TA, Soreq H. Function of alternative splicing. Gene. 2005;344:1-20.

59. Keating DJ, Chen C, Pritchard MA. Alzheimer's disease and endocytic dysfunction: clues from the down syndrome-related proteins, DSCR1 and ITSN1. Ageing Res Rev. 2006;5:388-401.

60. Kong XD, Liu N, Xu XJ. Bioinformatics analysis of biomarkers and transcriptional factor motifs in down syndrome. Braz J Med Biol Res. 2014;47:834-41.

61. Sharpe DA, Dixon K, Moghissi K. Endoscopic laser treatment for tracheal obstruction. Eur J Cardiothorac Surg. 1996;10:722-6.

62. Dergai M, Skrypkina I, Dergai O, Tsyba L, Novokhatska O, Filonenko V, Drobot L, Rynditch A. Identification and characterization of a novel mammalian isoform of the endocytic adaptor ITSN1. Gene. 2011;485:120-9.

63. Dergai M, Tsyba L, Dergai O, Zlatskii I, Skrypkina I, Kovalenko V, Rynditch A. Microexon-based regulation of ITSN1 and Src SH3 domains specificity relies on introduction of charged amino acids into the interaction interface. Biochem Biophys Res Commun. 2010;399:307-12. 
64. Kissil JL, Walmsley MJ, Hanlon L, Haigis KM, Bender Kim CF, Sweet-Cordero A, Eckman MS, Tuveson DA, Capobianco AJ, Tybulewicz VL, Jacks T. Requirement for Rac1 in a K-ras induced lung cancer in the mouse. Cancer Res. 2007:67:8089-94.

65. Archibald A, Mihai C, Macara IG, McCaffrey L. Oncogenic suppression of apoptosis uncovers a Rac1/JNK proliferation pathway activated by loss of Par3. Oncogene. 2015;34:3199-206.

66. Wang H, Teh MT, Ji Y, Patel V, Firouzabadian S, Patel AA, Gutkind JS, Yeudall WA. EPS8 upregulates FOXM1 expression, enhancing cell growth and motility. Carcinogenesis. 2010;31:1132-41.

67. Henson PM, Tuder RM. Apoptosis in the lung: induction, clearance and detection. Am J Physiol Lung Cell Mol Physiol. 2008;294:L601-11.

68. Predescu DN, Bardita C, Tandon R, Predescu SA. Intersectin-1s: an important regulator of cellular and molecular pathways in lung injury. Pulm Circ. 2013;3: 478-98.

69. Schmidt EP, Tuder RM. Role of apoptosis in amplifying inflammatory responses in lung diseases. J Cell Death. 2010;2010:41-53.

70. Di Fiore PP, De Camilli P. Endocytosis and signaling. An inseparable partnership. Cell. 2001;106:1-4.

71. Crosby LM, Waters CM. Epithelial repair mechanisms in the lung. Am J Physiol Lung Cell Mol Physiol. 2010;298:L715-31.

72. Derynck R, Zhang YE. Smad-dependent and Smad-independent pathways in TGF-beta family signalling. Nature. 2003;425:577-84.

73. Predescu S, Bardita C, Predescu D. New insights into the functions of intersectin-1s. Commun Integr Biol. 2015;8:e1034400.

74. Di Guglielmo GM, Le Roy C, Goodfellow AF, Wrana JL. Distinct endocytic pathways regulate TGF-beta receptor signalling and turnover. Nat Cell Biol. 2003:5:410-21.

75. McVey M, Tabuchi A, Kuebler WM. Microparticles and acute lung injury. Am J Physiol Lung Cell Mol Physiol. 2012;303:L364-81.

76. Predescu S, Shah TG, Qin S, Bardita C, Jeganathan N, Balk R, Predescu D. Two Runx1 isoforms determine the outcome in Ards. AJRCCM. 2017;195:A4783.

77. Lacroix R, Judicone C, Mooberry M, Boucekine M, Key NS, Dignat-George F, The ISSCW. Standardization of pre-analytical variables in plasma microparticle determination: results of the International Society on Thrombosis and Haemostasis SSC Collaborative workshop. J Thromb Haemost. 2013;11:1190-3.

78. Chen J, Li C, Chen L. The role of microvesicles derived from Mesenchymal stem cells in lung diseases. Biomed Res Int. 2015;2015:985814.

79. Hall JB, Kress JP. The burden of functional recovery from ARDS. N Engl J Med. 2011;364:1358-9.

80. Humbert M, Morrell NW, Archer SL, Stenmark KR, MacLean MR, Lang IM, Christman BW, Weir EK, Eickelberg O, Voelkel NF, Rabinovitch M. Cellular and molecular pathobiology of pulmonary arterial hypertension. J Am Coll Cardiol. 2004;43:13S-24S

81. Schermuly RT, Ghofrani HA, Wilkins MR, Grimminger F. Mechanisms of disease: pulmonary arterial hypertension. Nat Rev Cardiol. 2011;8:443-55.

82. Tuder RM, Marecki JC, Richter A, Fijalkowska I, Flores S. Pathology of pulmonary hypertension. Clin Chest Med. 2007;28:23-42. vii

83. Buzza MS, Hirst CE, Bird CH, Hosking P, McKendrick J, Bird PI. The granzyme B inhibitor, PI-9, is present in endothelial and mesothelial cells, suggesting that it protects bystander cells during immune responses. Cell Immunol. 2001;210:21-9.

84. Loeb CR, Harris JL, Craik CS. Granzyme B proteolyzes receptors important to proliferation and survival, tipping the balance toward apoptosis. J Biol Chem. 2006;281:28326-35.

85. Jeffery TK, Morrell NW. Molecular and cellular basis of pulmonary vascular remodeling in pulmonary hypertension. Prog Cardiovasc Dis. 2002;45:173-202.

86. Mortimer HJ, Peacock AJ, Kirk A, Welsh DJ. p38 MAP kinase: essential role in hypoxia-mediated human pulmonary artery fibroblast proliferation. Pulm Pharmacol Ther. 2007:20:718-25.

87. de Beer T, Carter RE, Lobel-Rice KE, Sorkin A, Overduin M. Structure and Asn-pro-Phe binding pocket of the Eps15 homology domain. Science. 1998; 281:1357-60.

88. Predescu S PM, Chen JDP. Targeted delivery of the NH2-terminal fragment of intersectin-1s triggers formation of complex plexiform lesions in mouse lungs. AJRCCM. 2015;191:A1936

\section{Submit your next manuscript to BioMed Central and we will help you at every step:}

- We accept pre-submission inquiries

- Our selector tool helps you to find the most relevant journal

- We provide round the clock customer support

- Convenient online submission

- Thorough peer review

- Inclusion in PubMed and all major indexing services

- Maximum visibility for your research

Submit your manuscript at www.biomedcentral.com/submit
) Biomed Central 